\title{
Nuclear Spins Detect Subtle Rotations
}

\section{A small device performs rotational measurements using nuclear spins in a diamond wafer, paving the way for microchip-size gyroscopes.}

\author{
By Mark Buchanan
}

$\Gamma$ uture technologies for unmanned and autonomous vehicles need precise gyroscopes for reliable flight and navigation, but these devices are typically too large to be suitable for lightweight microscopic electronics. Now researchers have demonstrated a tiny gyroscope that exploits the behavior of atomic nuclei in diamond [1]. Compared with previous diamond-based sensors, the device can measure slower rotational speeds-in a range (tens of degrees per second) that is relevant for aviation applications. The researchers expect that further development could lead to a new generation of commercially viable and ultrasensitive microchip gyroscopes.

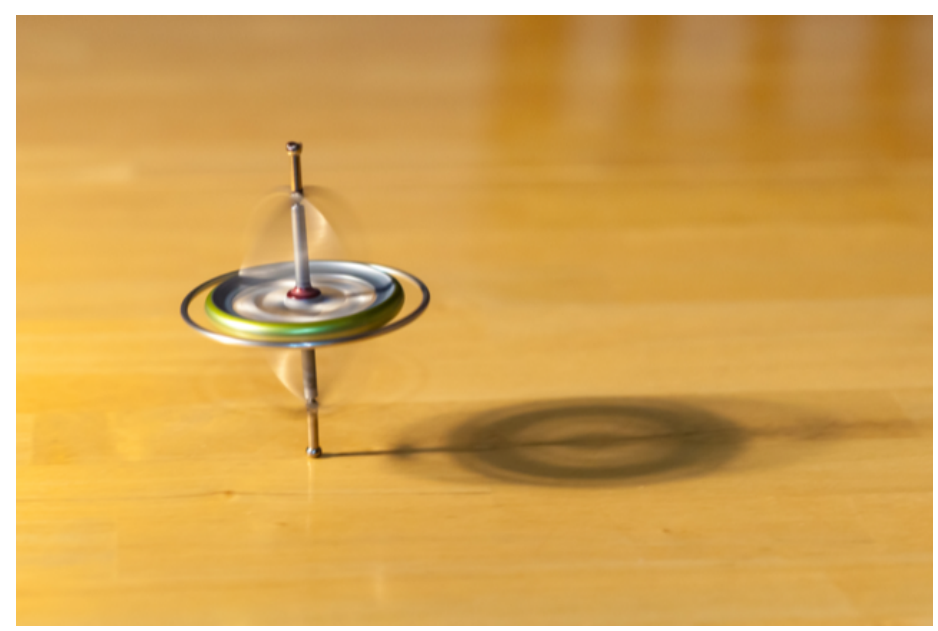

Hold that tilt. Like a toy gyroscope, the nuclear spins inside a diamond crystal maintain a stable orientation-which can be used to measure rotations.

Credit: Tom/stock.adobe.com
A gyroscope detects changes in the orientation of an object like an airplane. Today's most accurate gyroscopes employ laser light traveling on a closed path around a ring. But the accuracy of such devices depends on the area enclosed by the ring, so shrinking the gyroscopes would make them less reliable. Realizing small but still sensitive gyroscopes requires a different approach, and researchers have been developing alternatives based on exploiting the behavior of elementary particles that possess spin.

The spin state of a particle will stay fixed-like the spin axis of a toy gyroscope-unless acted on by some external force. Hence, a conceptually simple design for a gyroscope involves setting the spin of a particle in some state, leaving it alone, and then measuring the spin again. Any change would reveal a rotation of the object that encompasses the particle, which could be a flying vehicle, for example.

Making such a gyroscope work in practice, however, is challenging. Some researchers have been trying with atomic gases held in small traps, but collisions with walls tend to perturb the atomic spins. Using diamond avoids this problem. Diamond is made almost entirely of carbon atoms but also contains sites where a nitrogen atom replaces a carbon, while also leaving a nearby atomic vacancy in the lattice. These nitrogen vacancy (NV) centers have spins, both electronic and nuclear, that researchers have used to detect rotation (see Focus: Detecting the Rotation of a Quantum Spin) but not yet at the sensitivity level needed for a gyroscope.

Alexey Akimov of the Lebedev Physical Institute in Russia and colleagues have now demonstrated a gyroscope based on NV 
centers in a thin diamond wafer. Unlike previous work that focused on the spins of electrons or of carbon nuclei associated with NV centers, the researchers selected the spins of nitrogen nuclei as their rotation detectors. The nitrogen spins are less susceptible to perturbations than the other spins. However, the electron spins still proved useful in initializing and reading out the nuclear spins.

To demonstrate the operation of the gyroscope, the researchers placed their diamond wafer on a rotating platform embedded in a magnetic field. In such a field, the electron and nitrogen spins in an NV center are coupled through a light-mediated interaction. Taking advantage of this property, the team used a sequence of light pulses to set the nitrogen spins in an ensemble of NV centers into one spin state. They then let the spins evolve for 2 milliseconds before reading out the new spin state using a second sequence of pulses.

This read-out step took advantage of another convenient property of NV centers: the state of the electron spins-and hence the nuclear spins-can be inferred from the amount of light emitted from the NV centers. Rotating the platform slowly, at a speed of less than one revolution per second, the researchers measured the intensity of the emitted light and used this signal to calculate the platform rotation speed.

Besides providing setup and readout, the electron spins also played a secondary role in greatly improving the accuracy of the measurements, Akimov says. By monitoring the electron spins, the team could correct for a magnetic-field effect that causes an additional rotation of the nuclear spins. The team checked their gyroscope against a commercial microelectromechanical systems (MEMS) gyroscope and found good agreement. Akimov says the NV gyroscope could have an advantage over other gyroscopes in that it would not need constant recalibration.

Another advantage is that diamond crystals can be integrated into existing microchip technology more easily than gas-based devices can. "The solid-state nature of the sensor is a huge advantage," says physicist Dmitry Budker of the Johannes Gutenberg University in Germany, whose research group first suggested this approach in 2012. Akimov and colleagues are now miniaturizing their gyroscope so that it might eventually fit on a microchip. "Many things can be improved to make the device smaller," Akimov says.

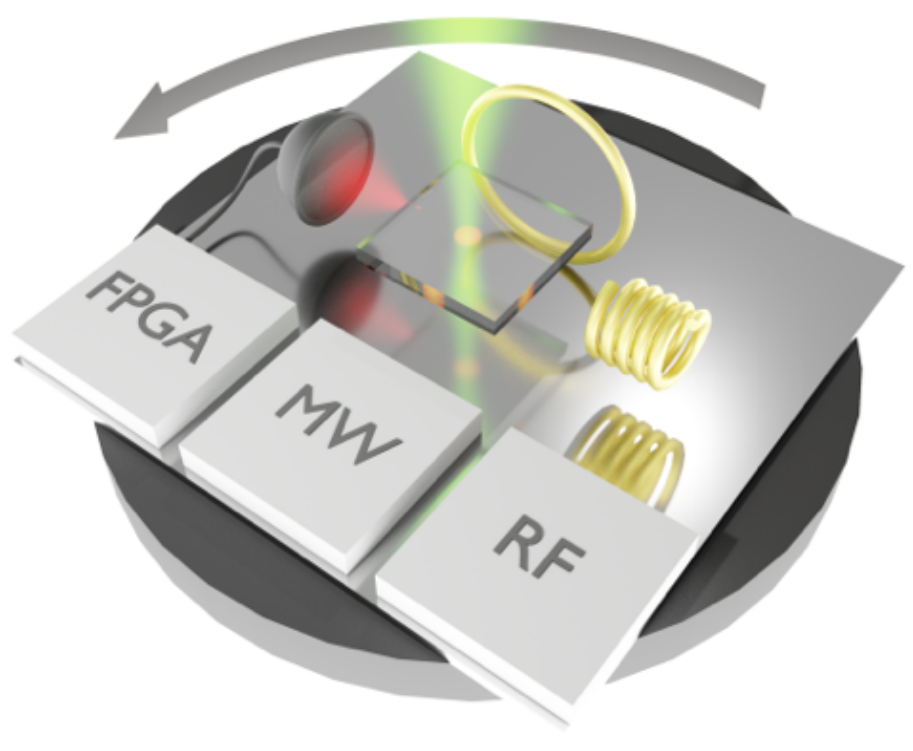

Spin cycle. A schematic of the nitrogen-vacancy (NV) gyroscope, placed on a rotating platform. The nitrogen nuclear spins in a diamond wafer are set by pulses from a laser (green), as well as inputs from microwave (MW) and radio-frequency (RF) components controlled by a field-programmable gate array (FPGA). After a free evolution period, the spin states are measured by reading out their emission.

Credit: V. V. Soshenko et al. [1]

Mark Buchanan is a freelance science writer who splits his time between Abergavenny, UK, and Notre Dame de Courson, France.

\section{REFERENCES}

1. V. V. Soshenko et al., "Nuclear spin gyroscope based on the nitrogen vacancy center in diamond," Phys. Rev. Lett. 126, 197702 (2021). 